QUEBEC AND MARITIME PROVINCES.

$A B$ YBERG, W. (AC.)

Riordon Pulp Corporation, St. Jovite Station, Quebec

BATT, C. A. (As.) Guif Pulp \& Paper Co., Clarke City, Quebec BEEDS, V. A. (AC.) Brown Corporation, Riviere du Loup, Quebec

BLAIR, R. J. (As.) Ormstown, Quebec

BOISVERT, GEORGE (AC.) Ontario Paper Co., Shelter Bay, Quebec

CAIN, M. V. (As.) Price Bros. \& Co., Chicoutimi, W., Quebec CAMPBELI, R. I. (AC.) Riordon Pulp Corporation, Montreal, Quebec

CAMPBELL, F. J. (AC.) Canada Paper Co., Winds or Mills, Quebec O CHAHOÚN, Jr., GEORGE (As.) Laurentide Co., Grand Mere, Quebec

CLARIDGE, B. E. (AC.) Hammermill Paper Co., Matane, Quebec COMMINS, M. J. (As.) Jas. D. Lacey \& Co. 921 Drummona Bìdg., Montreal, Quebec

COSENS, G. G. (AC.) Laurentide Co., Grand Piles, Quebec

DOWNS, H. A. (As.) Forestry Division, Laurentide Co., Grand Mere, Quebec

EARLE, T. W. (AC.) St. Lawrence Paper Mills, Three Rivers, Quebec 
EDGECOMBE, G. H. (A.C.)

Box 150, Three Rivers, Quebec

FENSOM, K. G. (As.)

700 University St., Montreal, Quebec

GAYLORD, F. A. (Ac.)

A. P. W. Paper Co., Sheet Harbour, N. S.

GILMOLR, J, $\mathbb{D}$, (AC.)

Anglo Canactian P. \& P. Co.,

II st. Peter st., Quebec, Quebec

GOSSELIN, C. (Ac.)

Quebec Forest Service, Berthierville, Quebec

GRAHAM, ANGUS (Ac.)

Quebec Forest Industries Assoc., Itd.,

126 st. Peter st., Quebec, Quebec

GREIG, DONALD (AC.) Brown Corp., 71 st. Peter st., Quebec, Quebec

HART, H. V。 (AC.)

637 Earnscliffe Ave., Montreal, Quebec

HOLIOWAY, E. S. (As.)

Hammermill Paper Co., Matane, Quebec

IRWIN, C. H. ( $\mathrm{AC}_{\mathrm{C}}$ )

Donnacona Paper Co.,

17 st. James St., Quebec, Quebec

JAGO, R. D. ( $\mathrm{Ac}$. $)$

Price Bros., \& Co., Chicoutimi, W., Quebec

JAMER, D. W. (AS.)

C/o James McIaren Co., Buckingham, Quebec

JENKINS, F. T. (AC.)

511 st. Catharines St., W., Montreal, Quebec

KIEFFER, HENRI, (As.)

Forest Protection Service, Quebec, Quebec

KIIBY, W. H. (As.)

Chief of Forest Protection, C. N. R., Montreal, Quebec

$\checkmark$ KYNOCH, W. (Ac.)

700 University St., Montreal, Quebec 
IINEKIN, M. G. (As.)

Canadian Intarnational Paper Co.,

Three Rivers, Quebec

IISTER, I. S. (As.)

Anticosti Corporation,

Anticosti Island, Quebec

IYONS, R. W. (Ac,)

Laurentide Co., Grand Mere, Quebec

MAHER, THOS. (A๑。)

$\mu$

2 Chauvear Ave., Quebec, Quebec

MARQUIS, I. J. D. (Ac.)

Giffard, Quebec

MILLER, G. I. (AC.)

Deptertment of Lands and Mines, Fredericton, N. B.

McCORMACK, W. W. (As.)

712 Union St., Fredericton, N. B.

MacGIBBON, F. J. (Ac.)

Dominion Frorest Products Laboratory,

700 University St., Montreal, Quebec

MeCOIM, W. F. (As.)

Price Bros. \& Co., Chicoutimi W., Quebec

NISBET, R. H. (Ac.)

Price Bros. \& Co., Quebec, Quebec

OTPY, C. D. (A. .)

Nashwaak Pulp \& Paper Co., St. John, N. B.

PERRIN, J. V. (As.)

Brown Corporation, 71 st. Peter st., Quebec, Quebec

PICHE, G. C. (Ac.)

Quebec Forest Service, Quebec, Quebec

PRINCE, G. H. (Ac.)

Department of Lands and Mines, Fredericton, N.B.

RICHARDS, E. S. (AS.)

710 Drummond Bldg., Montreal, Quebec 
ROY, HENRI (AC.)

28 Kitchener st., Quebec

ROY, J. A. (AC.)

57 scott St., Quebec, Quebec

SABBATON, F. A. (As.)

Laurentide Co,, Grand Mere, Quebec

SCHIERBECK, OTTO (AC.)

Provincial Forester, Halifax, N. S.

SCHUBERT, B. I. (AC.)

United Iast Co., Montreal, Quebec

SEABORNE, R. I. (As.)

Donnacona Paper Co., Quebec, Quebec

SMITH, KAARE (AC.)

Pejepscot Paper Co., Salmon River, N. B.

SORIUIS, HENRY (As.)

45 Maple Ave., Shawinigan Falls, Quebec

SUTHERLAND, J. W. (As.)

Canadian International Paper Co., Maniwaki, Quebec

TOWNSEND, C. R. (Ac.)

Iaurentide Co., Grand Mere, Quebec

WAITON, JOHN R. (AS.)

Price Bros. \& Co., Chicoutimi, Quebec

WEBB, PROF. H. P. (AC.)

Forestry Dept., University of N. B., Fredericton, N. B.

WEBB, I. S. (AC.)

278 Northumberland st., Fredericton, N.B.

WICKENDEN, H. R. (AC.)

Port Menier, inticosti Island, Quebec

WIISON, ELIWOOD (AC.)

Laurentide Co., Grand Mere, Quebec

WINEGAR, B. M. (AC.)

General Tie Agent, C. P. R., Montreal, Quebec 
WRIGHT, W. G. ( $\left.\mathrm{A}_{\mathrm{C}}\right)$

Price Bros. \& Co., Roberval, Quebec

ONT $\triangle R I O$.

$\triangle R D E N N E$, M. ( $\left.\mathrm{s}_{\text {. }}\right)$

Ontario Forestry Branch, Tweed, Ontario

ATKINSON, C. C. $(\Lambda \mathrm{c}$.

Woods Dept., Abitibi Power \& Paper Co., Iroquois Falls, Ontario

ATKINSON, W. F. V. (AC.)

81 Somerset St., Ottawa, Ontario

AUDEN, A. J. (As.) Fort William Paper Co.,

Union Bank Bldg., Fort William, Ontario

AVERY, B. F. (Ac.)

Spanish River Pulp \& Paper Mills,

Sault Ste Marie, Ontario

BAYLY, G. (As.)

Ontario Forestry Branch, Toronto, Ontario

BLACK, ROBSON (As.)

Canadian Forestry Association, Ottawa, Ontario

BLYTH, G. GER: Jrid (As.) 22 Hill st., Ottawa, Ontario

BRODIE, J. A. (AC.)

Ontario Forestry Branch, Tor onto, Ontario

BURK, A. H. (As.)

Spanish River Pulp \& Paper Mills, Sudbury, Ontario

BROWN, OSWALD W. (As.) Canadian International Paper Co.,

Sussex st., Ottawa, Ontario

GAMERON, D. R. (AC.)

Dominion Forestry Branch, Ottawa, Ontario

CANDY, R. H. ( Aco $\left._{0}\right)$ Dominion Forestry Branch, Ottawa, Ontario 
CARII.N, R.S. (AC)

Iroquois, ontario.

CASS, N.D. (AC.)

Dominion Forestry Branch, Ottawa, Ontario.

CONINELI, A.B. (AC.)

Ontario Forostry Branch, Sioux Lookout, Ontario.

COURTNAGE, R.i. (

Canadian International Paper Company,

Sussex St., Ottawa, Ontario.

CRAIG, R.D. (AC.)

Dominion Forestry Branch, Ottawa, Ontario.

CRAM, W.D. (AC.)

Ontario Forestry Branch, Kenora, Ontario.

CROSBIE, H.W. (i.c.)

Ontario Forestry Branch, Tweed, Ontario.

DALIYN, G.M. $\left(\Lambda c_{0}\right)$

Canadian Forestry Association,

51, sparks st., Ottawa, Ontario.

DAVIES, JOHN W. (As.)

Keewatin Lumber Company, Kenora, Ontario.

DAVIS, CLARK B. (AC.)

Woods Dept., Abitibi Power \& Paper Company, Iroquois Falls, Ontario.

DAVISON, E.S. (AS.)

Box 1013, Pembroke, Ontario.

DELAHEY, W.A. (AC.)

265, First Ave., Ottawe, Ontario.

DICKSON, J.R. (Ac.)

Dominion Forostry Branch, Ottawe, Ontario.

DRUCE, ERIC ( $\mathrm{Ac}$. )

Dominion Forestry Branch, Ottawe, Ontario.

DWIGHT, T.W. (AC.)

Faculty of Forestry, Univorsity of Toronto, Toronto, Ontario.

FINLAYSON, E.H. (Ac.)

Dominion Forestry Branch, Ottawa, Ontario. 
FOOTE, C.E. (AC.) Ontario Forestry Branch, Sudbury, Ontario.

GRAHAM, A.H. (L. .)

16, Fraser Blag., Ottawa, Ontario.

GREEIWOOD, W.B. (A.S.)

Canadian International Papor Company,

Suscex Stroet, Ottewa, Ontario.

HADEOW, W.R. (AC.)

Ontario Porestry Branch, Oba, Ontario.

HARRIS, AUSTIN S. (LS.)

16, Fraser Bldg., Ottawa, Onterio.

HORTON, F.H. (As.)

68, York St., St.Catharines, Ontario.

HOSIE, R.C. (As.)

Faculty of Forestry, University of Toronto, Toronto, Ontario.

HOWE, DR. C.D. (ic.)

Faculty of Forostry, University of Poronto, Tor onto, ontario.

HUGHSON, H.M. (Ac.)

Hope Chambers, Ottawa, Obtario:

JACOMBE, F.W.H. (Ac.)

Dominion Forestry Branch, Ottawa, Ontario.

JOHISTON, R.IT. (Ac.)

Ontario Forestry Branch, Poronto, Ontario.

KEISIT, N.IH. (As.)

Ontario Forestry Branch, Sioux Lookout, Ontario.

KIEFER, FRAICIS (Ac.)

Room 211, thalen Bldg., Port Arthur, Ontario.

KIIGSTON, G.A. (As.)

108, Argyle St., ottawa, ontario.

IAISE, G.R. (As.)

Ontario Forestry Branch, Toronto, Ontario, or 59, Grosvenor St., Toronto, Ontario. 
IEAVITT, CLYDE (AC.)

Board of Railway Commissioners, Ottawa, Ontario.

IESSARD, EUGENE (AC.)

C/O Canadian International Paper Company, Sussex St., Ottawa, Ontario.

LEWIS, R.G. (Ac.) Dominion Buagau of Statistics, Ottawa, Ontario.

IINTON, G.M. (Ac.)

Ontario Forestry Branch, Orono, Ontario.

MARRIME , I.C. (Ac.)

Midhurst Nursery, R.R. No. I, Anten Mills, Ontario.

MILIAR, PROFESSOR W.N. (AC.)

Faculty of Forestry, University of Toronto, Toronto, Ontario.

MILLS, C.R. (AC.)

Ontario Forestry Branch, Toronto, Ontario.

INULOY, G.A. (Ac.)

Dominion Forestry Branch, Ottawa, Ontario:

IGUNROE, D.J. (As.)

Woods Dept., Abitibi Power \& Paper Company, Iroquois Falls, Ontarior

MACDONALD, STUART C. (As.)

Woods Dept., Abitibi Pulp \& Paper Co., Ita., Iroquois Falls, ontario.

INACDOUGAII, F.A. (As.)

Ontario Forestry Branch, Sault Ste. Marie, Ontario.

IAACFAYDEN, C. (AC.)

Dominion Norestry Branch, Ottawa, Ontario.

IMCCALLUM, A.W. (Ac.)

Central Experimental Farm, Ottawa, Ontario.

MCDONALD, J.H. (AC.)

Ontario Forestry Branch, Pembroke, Ontario.

MCEWEN, P. (AC.)

Ontario Forestry Branch, Parry Sound, Ontario.

NEWMAN, F.S. (1..)

Ontario Forestry Branch, St. Williams, Ontario. 
PARSONS, H.H. (As.)

Ontario Forestry Branch, Toronto, Ontario.

PEPIER, W.A.E. (Ac.)

600 , Spadina Ave., Toronto, Ontario.

REID, I.H. ( AS.)

Ontario Forestry Branch, Toronto, Ontario.

RICHARDSON, A.H. (Ac.)

Ontario Forestry Branch, Toronto, Ontario.

ROBERTSON, W.M. (Ac.)

Dominion Forestry Branch, Ottawa, Ontario.

ROWE, C.A. (As.)

124, Flora St., Ottawa, Ontario.

SAUNDERS, E.G. (Ac.)

Dominion Forestry Branch, Ottawa, Ontario.

SCANDRETT, W.I. (AC,)

Box 242, Iond on, Ontario.

SCHANCHE, H.G. (AC.)

Woods Dept., Abitibi Power \& Paper Company, Iroquois Falis, ontario.

SHARPE, J.F. ( $(\mathrm{AC})$ Ontario Forestry Branch, Toronto, Ontario.

SIMMONS, J.F. I. (As.)

Ontario Forestry Branch, St. Williams, Ontario.

SIMPSON, E.R. (As.)

Keewatin Iumber Co., Kenora, Ontario.

SODERSTOIN, HERBERT R. (As.)

Woods Dept., Abitibi Power \& Paper Company, Iroquois Falls, Qntario.

SPARKS, N.C. (AS.)

Dominion Parks Branch, Ottawa, Ontario

STEWART, J.V. (As.)

22, Ferndale Ave., Tor onto, Ontario.

STEWART, K.A. (Ac.)

Ontario Forestry Branch, Sudbury, Ontario.

ase SWAINE, DR. J.N. (AC.)

Entomological Branch, ottawa, Ontario. 


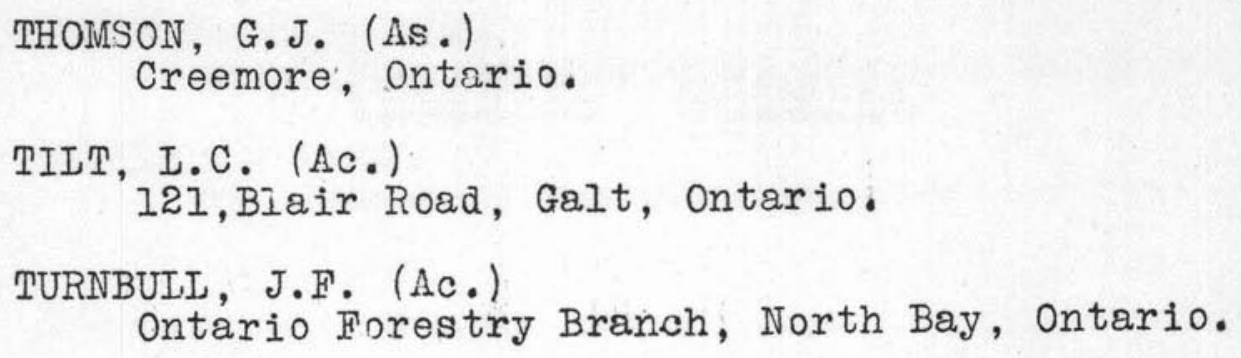

VENESS, J.C. (HC.) Dominion Forestry Branch, Ottawa, Ontario.

WESTLAND, C.E. (AC.) Ontario Forestry Branch,

WHITE, DR. J.H. (AC,) Faculty of Forestry, University of Toronto, Toronto, Ontario.

WIICOX, F.R.

Forestry Dept., Can. International Paper Company, Suwrex st., Ottawa, Ontario.

WILISON, W.E. (As.$)$

Woode Dopt., Abitibi Pulp \& Power Company, Iroquois Falls, ontario.

ZAVITZ, E.J. (AC.)

Ontario Forestry Branch, Toronto, Ontario.

\section{PRAIRIE PROVINCES.}

CHRISTIE, C.R. (Ac.)

Dominion Frost Branch, Annette, Saskatchewan.

CLARK, S.H. (AS.)

Entrance, Alberta.

COOPER, S.N. (As.)

Manitoba Paper Company, Pine Falls, Manitoba.

DE VEBER, H.A. (Ac.)

Dominion Forestry Branch, Rocky Mountain House, Alberta.

EISLER, H.P. (AC.)

Dominion Forestry Branch, Box 812, Prince Rupert, Saskatchewan. 
ELIIOT, G.B. (As.)

Dominion Forestry Branch, Rocky Mountain House, Alberta.

HALIIDAY, W.E.D. (AS.)

Dominion Forestry Branch, Greenbush, Saskatchewan.

HARRISON, J.D.E. (AS.)

Dominion Forestry Bervice, Customs Bldg., Winnipeg, Manitoba.

HOLIIAN, A.C. (A.s.)

1306, Eleventh Ave., W., Calgary, Alberta.

HOLMAN, H.I. (AC.)

Dominion Forestry Branch, Calgary, filberta.

HUESTIS, ERIC, S. (As.)

507 , loth st., West, Calgary, Alberta.

JACKSON, F.H.R. (LS.)

Dominion Forestry Branch,

Prince Rupert, Saskatchewan. ablent,

MARSHALI, E.J. (AS.) Dominion Forestry Branch, Kinistino, Saskatchewan.

MORSE, C.H. (Ac.)

Dominion Forestry Branch, Calgary, Alberta.

MORISON, MURRAY B, (As

Forestry Branch, Swan River, Manitoba.

MACDONALD, D.A. (AC.)

Dominion Forestry Branch, Winnipeg, Wanitoba.

MACFARLLINE, J.D.B. ( $\Lambda$ s.)

Dominion Forestry Branch, Winnipeg, Manitoba.

MACKENZIE, D.M. (AS.)

Dominion Forestry Branch, Coalspur, Alberta.

MACNEILI, WM. (As.)

Forestry Branch, Swan River, Manitoba.

IKCKENZIE, A.R. (AS.)

232, Victoria Ave., Brand on, Manitoba. 
PARKER, H.A. (AC.)

Dominion Forestry Branch, Maple Creek, Saskatchewan.

PHIPPS, G.W. (As.)

Manitoba Paper Company, Pine Falls, Manitoba.

PIKE, R.T. (As.)

Dominion Forestry Branch, Prairie River, Saskatchewan.

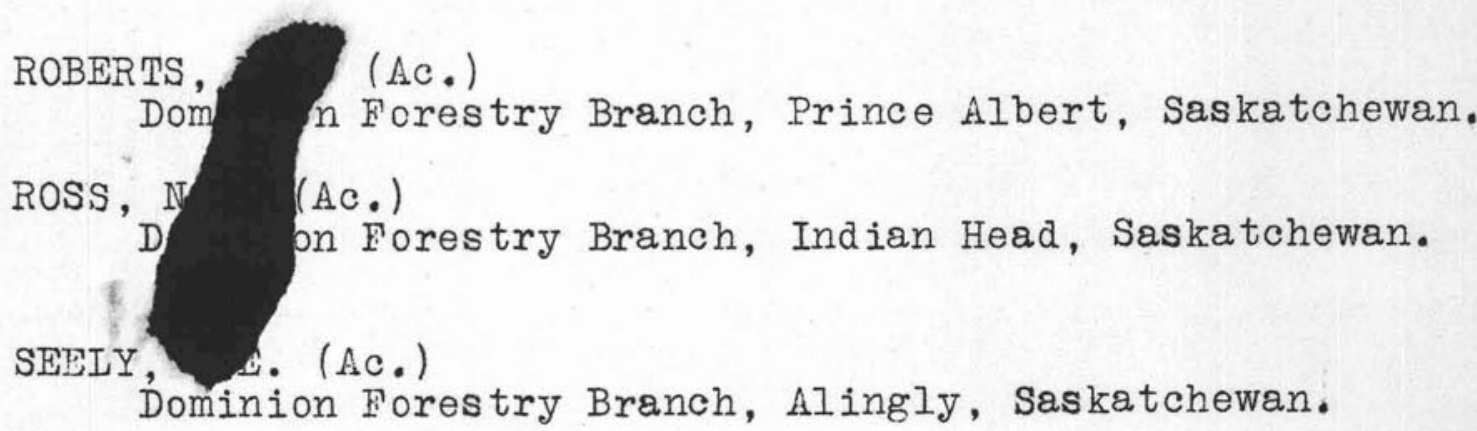

SMART, JAME'S (AS.)

Dominion Forestry Branch, Prince Albert, Saskatchewan.

STEVENSON, COI. H.I. (AC.)

Dominion Forestry Branch, Winnipeg, Manitoba.

TUNSTELI, GEORGE (AC.)

Dominion Forestry Branch, Winnipeg, Manitoba.

VAN CAMP, J.I. (AC.)

Dominion Forestry Branch, Pincher Creek, Alberta.

WATT, R.M. (AC.)

Dominion Forestry Branch, Dauphin, Manitoba.

\section{BRITISH COLUMBIA.}

ALEXANDER, J.F. (AS.)

B.C. Forest Branch, Victoria, B.C.

OR. ANDREWS, A.J. (Ac.)

Bloedel, B.C.

ARCHER, C.F. (As.)

B.C. Forest Branch, Nels on, B.C.

BARR, P.M. (As.)

B.C. Forest Branch, Victoria, B.C. 
BAXTER, H.H. (A.

513, Pacific Bldg., Vancouver, B.C.

BLAIR, M.P. (AS.) 702 , Belmont Blag., Victoria, B.C.

BONNEY, P.S. (AC.)

Woods Dept., Pacific Mills, Ocean Falls, B.C.

BROWN, I.I. (As.)

712, Metropilitan Bldg。, Vancouver, B.C.

BROWN, R.M. (AC.)

Dominion Forestry Branch, Kamloops, B.C.

CARLISIE, K.W. (As.)

2836, Ash St., Vancouver, B.C.

CAVERHIII, P.Z. (AC.)

B.C. Frorest Branch, Victoria, B.C.

CHRISTIE, PROFESSOR H.R. (AC.)

2575 , West 35 th St., Vancouver, B.C.

CIARKE, T.A. (AS.)

B.C. Forest Branch, Prince Rupert, B.C.

EADES, H.W. (AC.)

Forest Products Laboratories, University of B.C., Vancouver, B.C.

FLUIVERFELT, W.R. ( S. $\left._{\text {. }}\right)$

B.C. Forest Branch, Vancouver, B.C.

GARDINER, HAROLD (AS.)

513, Pacific Bldg., Vancouver, B.C.

GIBSOF, J.M. (Ac.)

B.C. Forest Branch, Victoria, B.C.

GIII, C.B. (As.) Dominion Forestry Branch, Swan River, B.C.

GRAINGER, M.A. (Ac.) Metropolitan Bldg., Vancouver, B.C.

GREGG, E.E. (AS.)

B.C. Forest Branch, Prince Rupert, B.C. 
HANSSON, (AS.)

C/O S.C. Hayden,

316, Winch Bldg., Vancouver, B.C.

HIBBERSON, R.W. (As.)

702, Belmont Bldg., Victoria, B.C.

JENKINS, J.H. (AC.)

Forest Products Laboratory, University of B.C., Vancouver, B.C.

JEWETP, C.A. (AC.)

Dominion Forestry Branch, Kamloops, B.C.

KAY, JAMES, (As.)

General Delivery, Vancouver, B.C.

KINGHORN, H.C. (AC.)

Box 20I, Cranbrook, B.C.

if KNAPP, PROHISGOR N.M. (AC.)

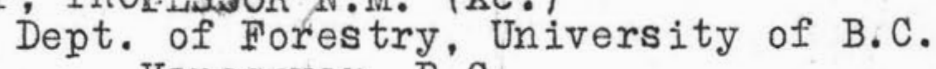

IYFORD, P.I. (Ac.)

Clark \& Lyford, Vancouver Block, Vancouver, B.C.

MANNING, E.C. (Ac.)

B.C. Forest Branch, Victoria, B.C.

MARIING, S.E. ( $\left.A s_{0}\right)$

B.C. Forest Branch, Box 290, Kamloops, B.C.

MELROSE, G.P. (AC.)

B.C. Forest Branch, Nelson, B.C.

MULHOLI.AND, F.D. (AC.)

B.C. Forest Branch, Victoria, B.C.

MACDONALD, F.A. (as)

Forest Branch, Victoria, B.C.

MACELHANNEY, T.A. (AC.) OS.

Forest Products Laboratories, University of B.C., Vancouver, B.C. 
MACMILIAN, H.R. (AC.)

712, Metropolitan Badg., Vancouver, B.C.

ORCHARD, C.D. (AC.)

B.C. Forest Branch, Prince George, B.C.

PARLOW, A.E. (AC.)

B.C. F'orest Service, Vancouver, B.C.

PERRY, R.S. (As,)

Forest Products Laboratory, University of B.C., Vancouver, B.C.

PICKFORD, A.E. (As.)

B.C. Forest Branch, Victoria, B.C.

PROWD, E.B. (Ac.)

B.c. Forest Branch, Victoria, B.c.

ST. CLAIR, R.C. (AC.)

B.C. Furest Branch, Vancouver, B.C.

STUART, R.V. (Ac.)

921, Metropolitan Bldg., Vancouver, B.C.

THRUPP, A.C. (AC.)

Dominion Forestry Branch, Kamloops, B.C.

VAN DUSEN, W. J. (Ac.)

712, Metropolitan Bldg., Vancouver, B.C.

WILKIISSON, T.H. (As.)

Canadian Fores tee Ass ociation, 521, Metropolitan Bldg., Vancouver, B.C.

\section{NON-RES IDENT.}

AYERS, B.K. (As.N)

35, Auburn St., Concord, N.H.

BENTLEY, A.W. (Ac.N.)

Newfoundland Power \& Paper Utilities Corporation, Cornerbrook, Newfoundland. 


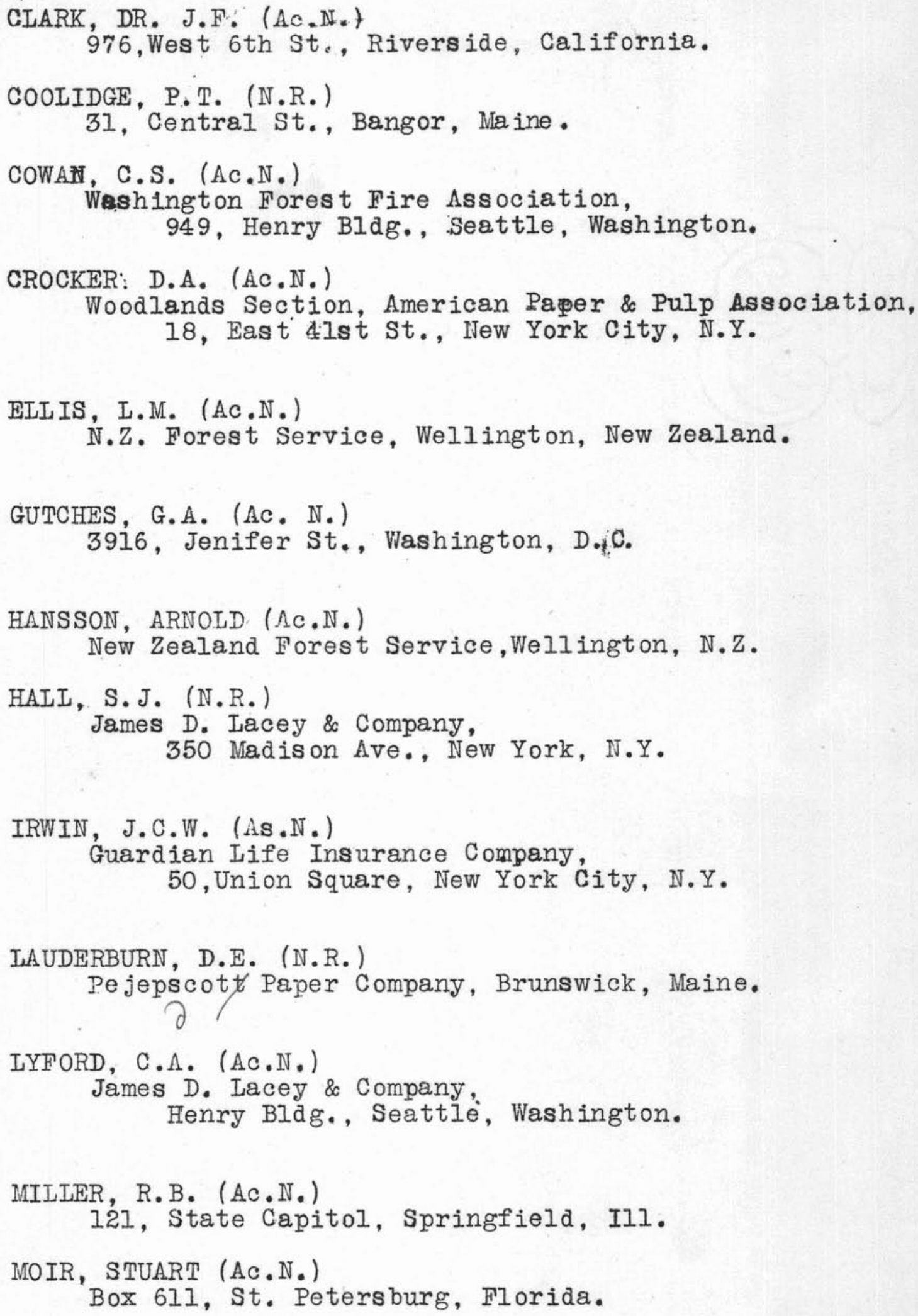




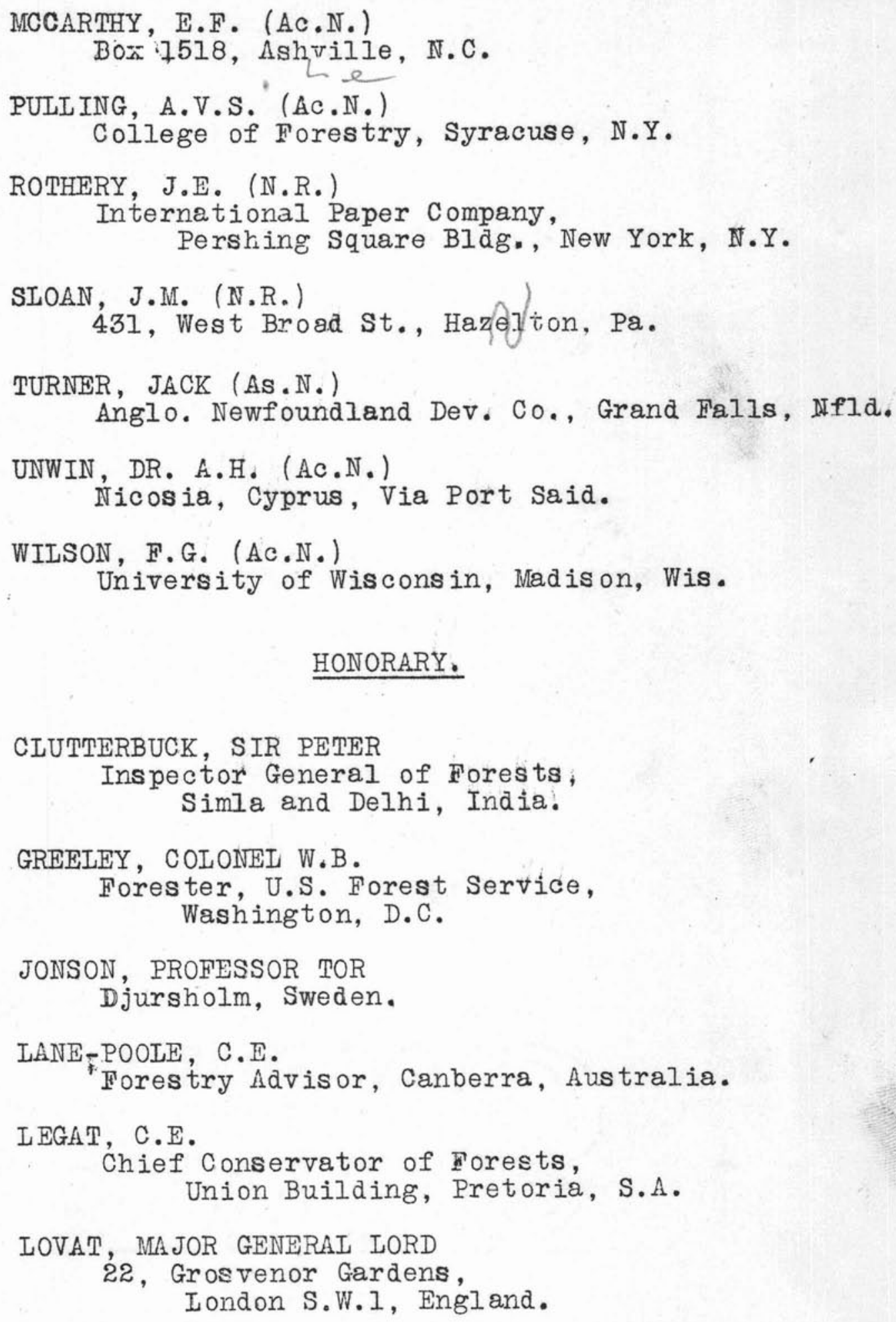

